\title{
Zero-order second harmonic generation from AlGaAs-on-insulator metasurfaces
}

\author{
Giuseppe Marino ${ }^{1}$, Carlo Gigli ${ }^{1}$, Davide Rocco ${ }^{2}$, Aristide Lemaître ${ }^{3}$, Ivan Favero ${ }^{1}$, \\ Costantino De Angelis ${ }^{2}$, and Giuseppe Leo ${ }^{1}$
}

${ }^{1}$ Matériaux et Phénomènes Quantiques, Université Paris Diderot \& CNRS, 10 rue Alice Domon et Léonie Duquet, 75013 Paris, France

${ }^{2}$ Department of Information Engineering, Università degli Studi di Brescia and INO-CNR, Via Branze 38, 25123 Brescia, Italy

${ }^{3}$ Centre de Nanosciences et de Nanotechnologies, CNRS - Université Paris-Saclay, Palaiseau, France

\section{Contents}

I. Experimental Setup

II. Extended measurements

III. Extended numerical simulations 


\section{Experimental Setup}

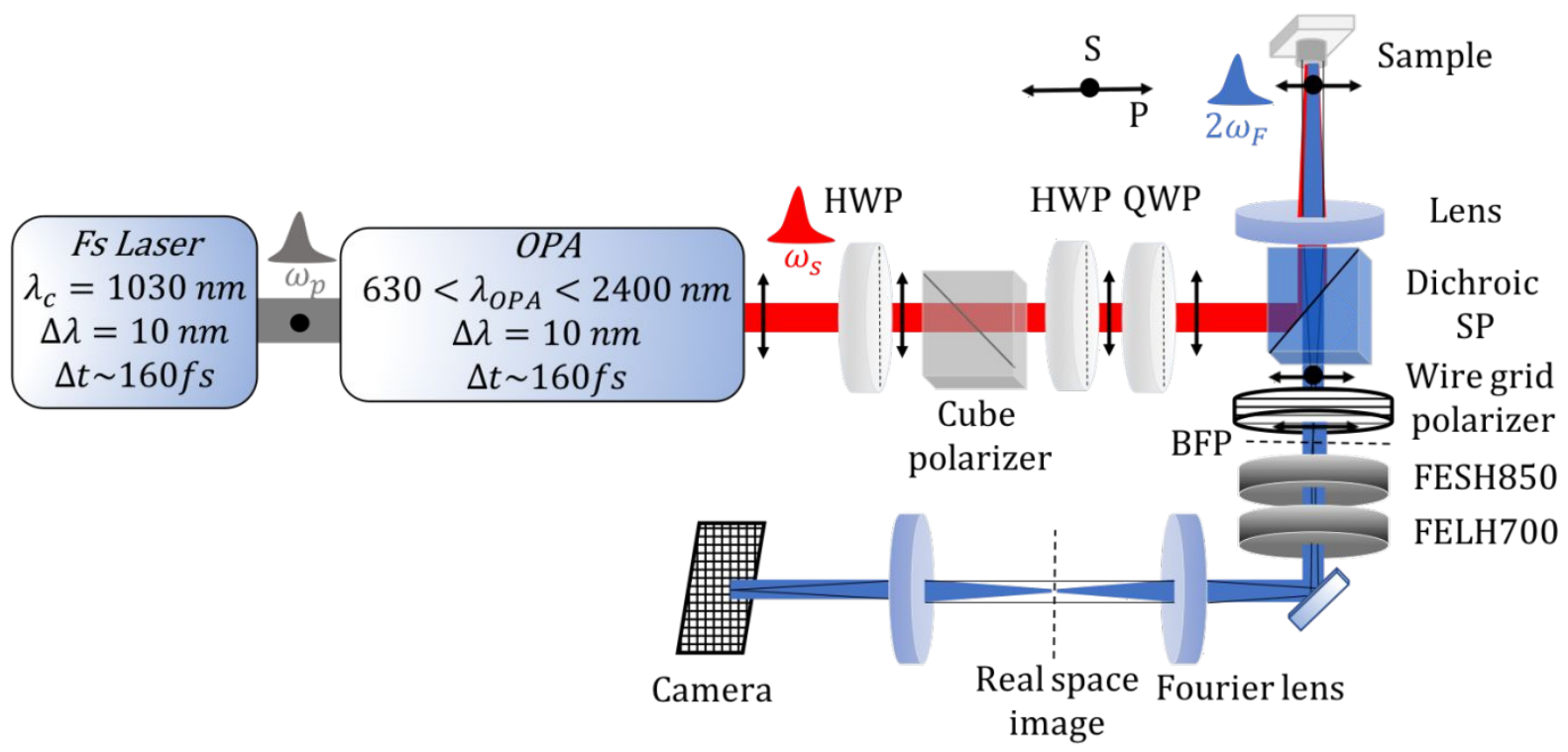

Figure S1. Schematic representation of the illumination and detection geometry. Input beam is focused by a $0.5 \mathrm{NA}$ lens, while SHG collected in reflection from the same lens is limited to a $0.1 \mathrm{NA}$ by our imaging system. 


\section{Extended measurements}
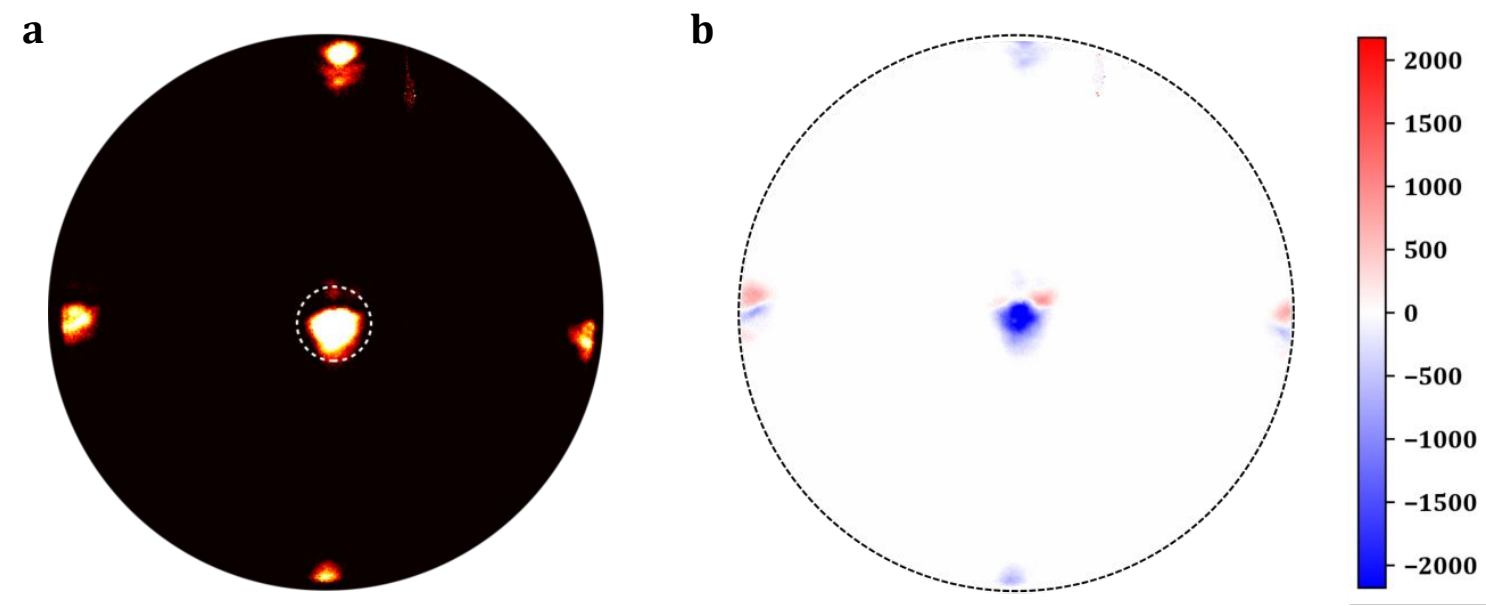

Figure S2. (a) Measured unpolarized radiation pattern of reflected SHG from AlGaAs-on-AlOx metasurface. The corresponding experimental layout is similar to Figure S1, except that the pump beam waist on the rear pupil of the 0.8 NA objective (no lens in this case) is reduced, resulting in a larger beam spot size. The first orders are partially cut by the objective NA. The white dashed line including the zero order corresponds to a $N A=0.1$. The relative intensity between the zero and first orders depends on the overlap between the array factor and the radiation pattern of the isolated nanoresonator. With regard to the case of Figure 1c, this different detection system (higher NA and magnification) lowers the resolution within a $N A=0.1$, to the point that two distinct lobes of Figure 1c are here merged. (b) Measured polarized radiation pattern of reflected SHG from AlGaAs-onAlOx metasurface. Co-polarized and cross-polarized signals are shown in blue and red, respectively. $90 \%$ of SHG signal is cross-polarized. 


\section{Extended numerical simulations}

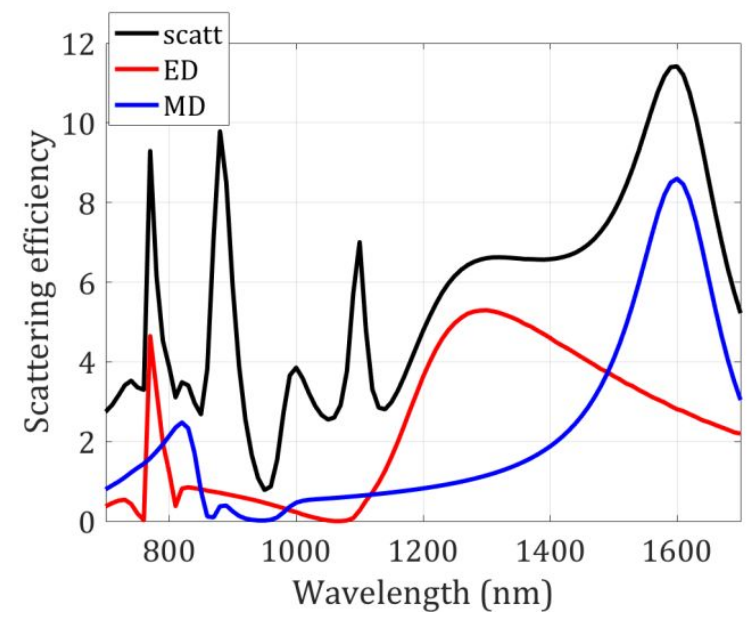

Figure S3. Scattering efficiency and multipolar decomposition associated to the single nanoantenna with $h=400 \mathrm{~nm}, R=200 \mathrm{~nm}$ (ED: electric dipole; MD: magnetic dipole).

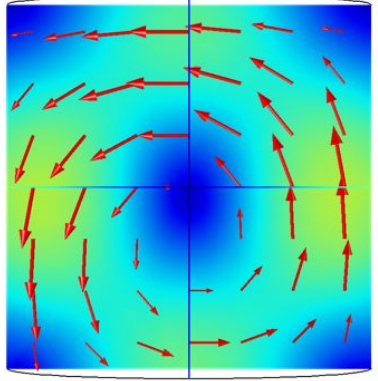

a
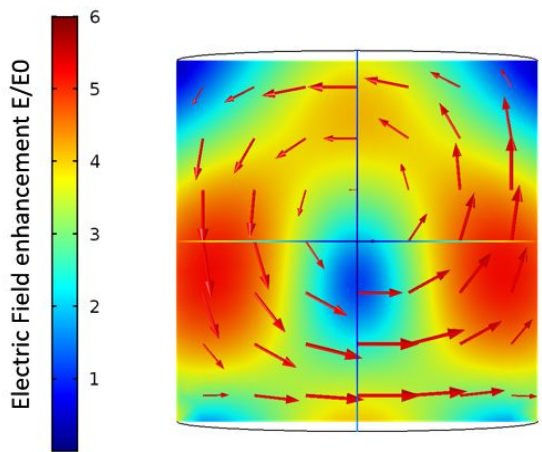

b

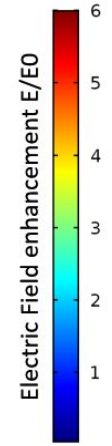

Figure S4. Electric field enhancement for a nanocylinder with radius $200 \mathrm{~nm}$, respectively isolated (a) and in the metasurface configuration with periodicity $1025 \mathrm{~nm}$ (b). The red arrows indicate the electric field vector (in-plane components). 